\section{CASE REPORT}

\author{
J.G. Seol \\ L.A. Loevner \\ B.W. O'Malley, Jr \\ M.S. Grady
}

\title{
Inflammatory Pseudotumor of the Trigeminal Nerve: A Neoplastic Mimic You Do Not Want to Miss
}

SUMMARY: Inflammatory pseudotumor is a rare non-neoplastic mass that may clinically and radiologically mimic a spectrum of benign and malignant neoplasms. It is uncommon in the head and neck and particularly rare at the skull base. We present a case of pseudotumor originating from the trigeminal nerve in a patient who presented with headache and facial numbness. A high index of suspicion is necessary to diagnose this benign but locally aggressive entity.

nflammatory pseudotumor (IPT) is uncommon and may be indistinguishable from a spectrum of benign and malignant tumors. Originally reported in the lung, IPT has now been described in nearly every anatomic site. ${ }^{1}$ In the head and neck, it is uncommon but most often affects the orbit. Skull base pseudotumor is rare and tends to behave aggressively, often mimicking a neoplasm. In this article, we discuss a rare case of IPT isolated to the skull base and originating from the trigeminal nerve.

\section{Case Report}

An otherwise healthy 24-year-old woman presented with left-sided headache and facial numbness for 6 months. She denied tinnitus, difficulty hearing, or dysphagia. Physical examination revealed left periorbital swelling and hypesthesia in areas supplied by the trigeminal nerve. Findings of the remainder of her neurologic examination were unremarkable. Outside imaging revealed a skull base mass, which was biopsied endoscopically. Histology showed inflammation, and the patient was placed on corticosteroids. When her symptoms did not improve, she was admitted for pain control.

Repeat MR and CT imaging revealed an expansile enhancing mass in the left infratemporal fossa with extension to the skull base and along all divisions of the trigeminal nerve, with intracranial extension and cavernous sinus invasion (Fig $1 A-E$ ). There was extension along the nerve in the prepontine cistern with compression and edema of the pons. Remodeling of the left petrous apex and expansion of the foramen ovale and infraorbital canal were identified. The mass was hypo- to isointense relative to brain on $\mathrm{T} 2$-weighted images, and there was no restricted diffusion.

Laboratory work-up included complete blood cell count and basic metabolic panel, which had normal results. Further work-up of an inflammatory process, such as erythrocyte sedimentation rate, was

Received March 2, 2009; accepted after revision March 23

From the Departments of Radiology (J.G.S., L.A.L.), Otorhinolaryngology (L.A.L., B.W.O.), and Neurosurgery (L.A.L., M.S.G.), University of Pennsylvania, Philadelphia, Pa.

Julia G. Seol is a recipient of the National Institute of Diabetes and Digestive and Kidney Diseases (NIDDK) short-term research training grant for medical students (grant number 5-T35-DK-60441-08)

Please address correspondence to Laurie A. Loevner, MD, Department of Radiology, Hospital of the University of Pennsylvania, 2 Dulles Building, 3400 Spruce St, Philadelphia, PA 19104; e-mail: laurie.loevner@uphs.upenn.edu

\section{Indicates open access to non-subscribers at www.ajnr.org}

not performed because the history of steroid-unresponsiveness suggested that the condition was not inflammatory in etiology.

The patient underwent a retromastoid suboccipital craniotomy. Intraoperatively, the mass engulfed the trigeminal nerve, and the portion compressing the brain stem was resected. Histologic evaluation with S100 and neurofilament immunostains showed nerve fibers disrupted and surrounded by an inflammatory infiltrate of B and T lymphocytes, plasma cells, and macrophages. There was no evidence of atypia or neoplasia. Stains were negative for bacteria, fungi, and acidfast bacilli. Tests for Epstein-Barr Virus (EBV) and herpes and varicella zoster viruses were negative. The final diagnosis was IPT.

The patient had no complications and was discharged on oral steroids. She had improvement in facial sensation and headache severity. Imaging performed 8 weeks following surgery while she was on steroids revealed significant reduction in the size of the mass (Fig $1 F,-G)$.

\section{Discussion}

Knowledge of head and neck pseudotumor is largely based on experience with orbital pseudotumor, one of the most common presentations of IPT. ${ }^{1}$ In the head and neck, pseudotumor rarely involves the skull base and even less commonly emanates from a cranial nerve. Our review of reported cases indicates that skull base IPT occurs primarily in adults, shows no predilection for sex, and behaves more aggressively than other head and neck pseudotumors. ${ }^{2-7}$

Skull base IPT typically presents with local pain, swelling, and/or cranial nerve palsies, as in our patient. Cranial nerve palsies are most often due to neural compression from the mass. Extensive primary neural involvement has only been reported in isolated cases. ${ }^{5,6}$ Systemic symptoms such as fever or weight loss are uncommon in head and neck IPT, though they occur in pseudotumors affecting other sites. ${ }^{1,8}$

Imaging frequently reveals a moderately enhancing softtissue mass. MR imaging findings include a mass that is iso- to hypointense relative to brain on T2-weighted images. ${ }^{1-7}$ The presence of marked T2 hypointensity can be useful in suggesting IPT rather than other skull base neoplasms that typically are iso- to hyperintense on T2-weighted images. In contrast to orbital disease, extraorbital IPT often causes bone sclerosis, erosion, and remodeling. ${ }^{1-3,7}$ In addition to these imaging features, our patient also had extensive perineural tracking along the trigeminal nerve. 

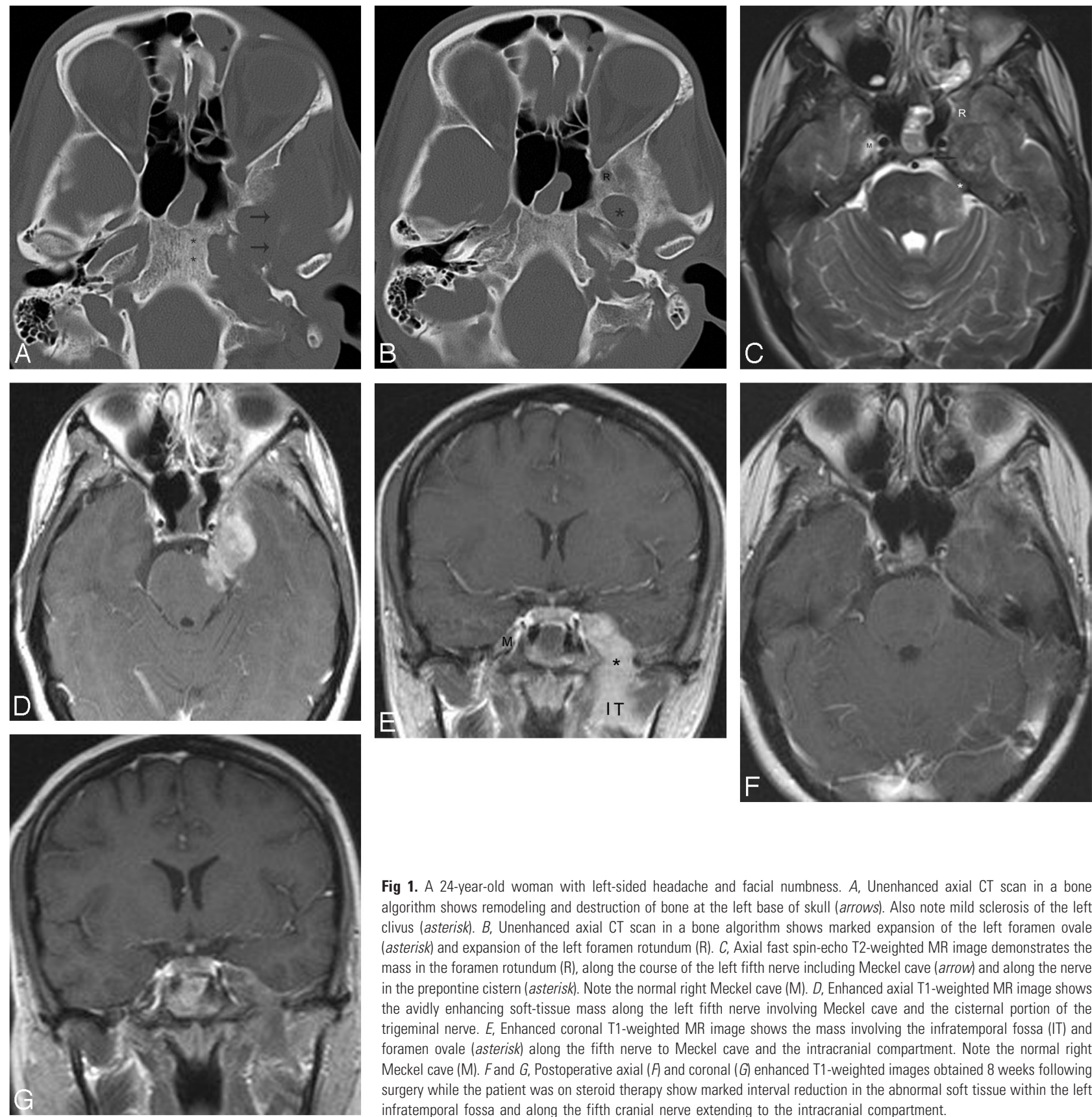

Fig 1. A 24-year-old woman with left-sided headache and facial numbness. $A$, Unenhanced axial CT scan in a bone algorithm shows remodeling and destruction of bone at the left base of skull (arrows). Also note mild sclerosis of the left clivus (asterisk). B, Unenhanced axial CT scan in a bone algorithm shows marked expansion of the left foramen ovale (asterisk) and expansion of the left foramen rotundum (R). C, Axial fast spin-echo T2-weighted MR image demonstrates the mass in the foramen rotundum (R), along the course of the left fifth nerve including Meckel cave (arrow) and along the nerve in the prepontine cistern (asterisk). Note the normal right Meckel cave (M). D, Enhanced axial T1-weighted MR image shows the avidly enhancing soft-tissue mass along the left fifth nerve involving Meckel cave and the cisternal portion of the trigeminal nerve. E, Enhanced coronal T1-weighted MR image shows the mass involving the infratemporal fossa (IT) and foramen ovale (asterisk) along the fifth nerve to Meckel cave and the intracranial compartment. Note the normal right Meckel cave (M). Fand G, Postoperative axial $(F$ and coronal $(G)$ enhanced T1-weighted images obtained 8 weeks following surgery while the patient was on steroid therapy show marked interval reduction in the abnormal soft tissue within the left infratemporal fossa and along the fifth cranial nerve extending to the intracranial compartment.

Our patient's presentation and imaging findings were thought to be most consistent with a neoplasm such as schwannoma, though perineural involvement from other neoplastic processes was considered. Lymphoma, sarcoidosis, and inflammatory conditions, which may share similar MR imaging findings with IPT, were initial considerations. However, the mass did not demonstrate restricted diffusion, the patient had no systemic symptoms, and her condition was steroid-unresponsive, so these were considered to be less likely.

Pseudotumor has no pathognomonic imaging characteristics, and biopsy is required for diagnosis. The unifying features of IPT are the absence of neoplastic cells or microorganisms and the presence of inflammatory cells with fibrosis. Lymphocytes, plasma cells, or eosinophils may predominate in the inflammatory component, or it may be heterogeneous in composition. ${ }^{4,5}$ The hypointensity seen on T2-weighted images likely reflects a combination of fibrosis and attenuated cellularity.

The etiology of pseudotumor is unclear but is thought to be infectious or autoimmune in nature. Elevated serum inflammatory markers are frequently noted, ${ }^{4}$ and it has been suggested that IPT may be the result of an immune response to an underlying infection. Associations with EBV and other agents have been suggested, ${ }^{5,8}$ though microorganisms are rarely detected. ${ }^{2-4}$

Jung et $\mathrm{a}^{5}$ described another case of pseudotumor derived from the trigeminal nerve that demonstrated histopathologic differences compared with our case, despite similar clinical presentations and anatomic and cellular origin. The authors 
reported that the tumor cells showed immunopositivity for leukocyte common antigen (LCA) and immunonegativity for S100. Both LCA and S100 are found in cells of nervous tissue origin, and the immunopositivity of our specimen for S100 and theirs for LCA helped confirm the trigeminal nerve origin of our cases. In contrast, their case showed immunopositivity for EBV, whereas our specimen was negative for markers of infection. However, the significance of this difference is unknown, especially given the paucity of information regarding skull base IPT arising from nerve; further study of additional cases is certainly needed to evaluate the postinfectious hypothesis.

Surgical resection of skull base IPT has generally proved to be curative. ${ }^{2,5,7}$ In contrast to pseudotumors of the orbit, extraorbital skull base IPT responds poorly to steroids ${ }^{3,4}$ and may require additional treatment. Radiation therapy has been used in patients with orbital IPT who cannot tolerate or fail steroid treatment, ${ }^{1}$ but similar success has not been demonstrated in the limited reported cases of skull base IPT. ${ }^{4}$ In our case, the patient was initially steroid unresponsive, but she had significant clinical and radiologic improvement following surgical decompression and subsequent steroid therapy.

\section{References}

1. Narla LD, Newman B, Spottswod SS, et al. Inflammatory pseudotumor. Radiographics 2003;23:719-29

2. De Vuysere S, Hermans R, Sciot R, et al. Extraorbital inflammatory pseudotumor of the head and neck: CT and MR findings in three patients. AJNR Am J Neuroradiol 1999;20:1133-39

3. Han MH, Chi JG, Kim MS, et al. Fibrosing inflammatory pseudotumors involving the skull base: MR and CT manifestations with histopathologic comparison. AJNR Am J Neuroradiol. 1996;17:515-21

4. Lee DK, Cho YS, Hong SH, et al. Inflammatory pseudotumor involving the skull base: response to steroid and radiation therapy. Otolaryngol Head Neck Surg 2006;135:144-48

5. Jung T, Jung S, Lee M, et al. Hemorrhagic intracranial inflammatory pseudotumor originating from the trigeminal nerve: a case report. J Neurooncol 2006;76:139-42

6. Yanagihara N, Segoe M, Gyo K, et al. Inflammatory pseudotumor of the facial nerve as a cause of recurrent facial palsy: case report. Am J Otol 1991;12:199-202

7. Strasnick B, Vaughan A. Inflammatory pseudotumor of the temporal bone: a case series. Skull Base 2008;18:49-52

8. Arber DA, Kamel OW, van de Rijn M, et al. Frequent presence of the Epstein-Barr virus in inflammatory pseudotumor. Hum Pathol 1995;26: 1093-98 